\title{
Survey of Hypoglycemia in Elderly People With Type 2 Diabetes Mellitus in Japan
}

\author{
Masahiro Fukuda ${ }^{\text {a, f, g, Kunihiro Doi }}{ }^{\text {b, f, }}$, Masahiro Sugawara ${ }^{\text {c, f, }}$, Yoshikazu Naka ${ }^{\text {d, f }}$, \\ Kouichi Mochizukie, $f$
}

\begin{abstract}
Background: The number of elderly type 2 diabetes mellitus (T2DM) patients in Japan is increasing continuously. Hypoglycemia is a significant issue in their treatment. However, the actual situation and related details of their hypoglycemia remain unclear. In order to elucidate them, the Japan Physicians Association conducted a largescale questionnaire survey for physicians and their outpatients all over Japan.
\end{abstract}

Methods: Targeted elderly T2DM outpatients were 65 years old or older in 2011. Specialized questionnaire survey forms were distributed to both of physicians and patients. The forms for physicians included questions whether patient had hypoglycemia in the last 1 month or 1 year; those for patients included whether they experienced it in the same durations and any of the 28 symptoms that are suggestive of hypoglycemia or pertaining to geriatric syndrome in the last 1 month, as well as questions about knowledge regarding hypoglycemia. We analyzed associations between hypoglycemia and the symptoms, and between hypoglycemia and medications.

Results: Of 15,892 T2DM patients (age, 74.2 \pm 6.3 years; diabetes duration, $12.8 \pm 8.9$ years; HbA1c, $7.0 \pm 1.0 \%$ ), dipeptidyl peptidase-4 inhibitor (DPP-4i) was the most prescribed medication among all oral hypoglycemic agents (OHAs). The frequencies of hypoglycemia in the last 1 month recognized by physicians and experienced by patients were $7.8 \%$ and $10.4 \%(\mathrm{P}<0.0001)$, and in the last 1 year were $15.5 \%$ and $21.1 \%$ respectively $(\mathrm{P}<0.0001)$. The most common symptom was "weakness, fatigue/feeling languid" and the majority of all pa-

Manuscript accepted for publication October 01, 2015

aFukuda Clinic, 2F Shin Osaka Brick Building, 1-6-1 Miyahara, Yodogawaku, Osaka-shi, Osaka 532-0003, Japan

bDoi Clinic, 1-54 Todou Aramaki, Uji-shi, Kyoto 611-0013, Japan

'Sugawara Clinic, 3-9-16 Shakujiimachi, Nerima-ku, Tokyo 177-0041, Japan

${ }^{\mathrm{d} T o m e i-A t s u g i}$ Hospital, 232 Funako, Atsugi-shi, Kanagawa 243-8571, Japan

eMochizuki Naika Clinic, 1F Katsura Heights, 4-5 Aioicho, Itabashi-ku, Tokyo 174-0044, Japan

${ }_{\mathrm{f}}$ Member of the Japan Physicians Association.

gCorresponding Author: Masahiro Fukuda, Fukuda Clinic, 2F Shin Osaka Brick Building, 1-6-1 Miyahara, Yodogawa-ku, Osaka-shi, Osaka 532-0003, Japan. Email: fukuda@drmog.jp

doi: http://dx.doi.org/10.14740/jocmr2340w tients reported neuroglycopenic or autonomic symptoms. Regarding monotherapy, hypoglycemia was observed in $32.7 \%$ of the patients with insulin, $4 \%$ in sulfonylurea (SU), 3.8\% in glinide, and 3.5\% in pioglitazone. The questions asking knowledge about hypoglycemia revealed that SU or insulin users had significantly more knowledge of hypoglycemia than others $(\mathrm{P}<0.001)$; however, $63 \%$ of patients using insulin, and $31 \%$ of patients using SU always carried glucose or a similar medication with them.

Conclusions: The present study suggested two types of "hidden hypoglycemia", one is that physicians did not detect and the other one is that patients were not aware. It is vital that physicians strive to prevent hypoglycemia by paying closer attention to symptoms of "hidden hypoglycemia" in their elderly patients.

Keywords: Hypoglycemia; Hypoglycemic symptom; Hidden hypoglycemia; Knowledge of hypoglycemia; T2DM; Elderly; Medication; Countermeasure

\section{Introduction}

The number of patients with type 2 diabetes mellitus (T2DM) in Japan has been increasing. According to the International Diabetes Federation (IDF) Diabetes Atlas [1], the number of patients with diabetes aging $20-79$ years in 2014 was 7.21 million in Japan. Furthermore, it is estimated that additional 3.89 million undiagnosed patients with suspected diabetes exist. Hence, Japan is ranked 10th worldwide in terms of its diabetic population. In particular, the population is progressively aging in Japan [2]; thus, the proportion of elderly individuals in patients with diabetes is higher compared with other countries. Moreover, according to the 2014 National Health and Nutrition Survey, increasing patients in those with diabetes were the elderly patients in Japan, and it will continue to increase.

Hypoglycemia is a significant issue in treatment of diabetes for the elderly patients. Hypoglycemia worsens quality of life (QOL) and reduces quality-adjusted life years (QALY) [3]. In particular, elderly individuals also experience symptoms caused by aging as follows: decreased activities of daily living, lowered physiological function of the liver and kidneys, and decreased cognitive function. Thus, elderly patients 


\begin{tabular}{|c|c|c|c|c|c|}
\hline \multicolumn{6}{|c|}{$\begin{array}{l}\text { Do you have any symptoms as described below } \\
\text { in the last one month (multiple choices allowed) }\end{array}$} \\
\hline$\square$ cold sweat & \multicolumn{2}{|c|}{$\square$ palpitations } & \multicolumn{2}{|l|}{$\begin{array}{l}\square \text { weakness, fatigue/ } \\
\text { feeling languid }\end{array}$} & $\square$ tremulousness \\
\hline $\begin{array}{l}\square \text { dizziness/ } \\
\text { lightheadedness }\end{array}$ & \multicolumn{2}{|c|}{$\square$ headche } & \multicolumn{2}{|l|}{ 口lethargy } & $\square$ frequent yawning \\
\hline \multicolumn{3}{|l|}{$\square$ fasting irritation } & \multicolumn{2}{|l|}{$\square$ speech difficulty } & $\square$ blurred vision \\
\hline$\square$ coldness & \multicolumn{2}{|c|}{$\square$ nausea } & \multicolumn{3}{|l|}{$\square$ hunger } \\
\hline \multicolumn{6}{|l|}{$\square$ hyperphagia } \\
\hline \multicolumn{2}{|l|}{$\square$ loss of hunger/fullness } & \multicolumn{2}{|c|}{$\square$ epigastric distress } & \multicolumn{2}{|c|}{$\begin{array}{l}\square \text { cognitive dysfunction/ } \\
\text { forgetfulness }\end{array}$} \\
\hline \multicolumn{2}{|l|}{$\square$ cramps } & \multicolumn{2}{|c|}{$\square$ constipation } & \multicolumn{2}{|c|}{$\square$ back pain } \\
\hline \multicolumn{2}{|l|}{$\square$ frequent urination } & \multicolumn{2}{|c|}{$\square$ thirsty } & \multicolumn{2}{|c|}{$\square$ numbness in hands } \\
\hline \multicolumn{2}{|l|}{$\square$ foot numbness } & \multicolumn{2}{|c|}{ ㅁleg cramp } & \multicolumn{2}{|c|}{$\square$ swelling of foot } \\
\hline \multicolumn{4}{|c|}{$\square$ abnormal sense of the tip of a foot } & & \\
\hline \multicolumn{6}{|c|}{$\begin{array}{l}\text { (1) For doctors } \\
\text { - Assessing patients' hypoglycemia in the last one month }\end{array}$} \\
\hline \multicolumn{6}{|c|}{$\begin{array}{l}\text { (2) For patients } \\
\text { - Reporting experiencing hypoglycemia in the last one month }\end{array}$} \\
\hline \multicolumn{6}{|c|}{$\begin{array}{l}\text { (3) For patients: symptoms experienced in the last one month } \\
\text { - } 15 \text { symptoms related to hypoglycemia (upper) }\end{array}$} \\
\hline
\end{tabular}

Figure 1. Questionnaire of hypoglycemia. The items were made in reference to a chapter of hypoglycemia in MEDSi Joslin Diabetes.

are more likely to develop iatrogenic hypoglycemia than nonelderly patients. In addition, hypoglycemic symptoms change with age and those symptoms are often atypical expression in elderly individuals [4]; therefore, treatment for their hypoglycemia tends to be delayed and increasing disease severity. According to the report regarding severe hypoglycemia such as hypoglycemic coma in emergency visit [5], three major factors are advanced age, renal function degeneracy, and sulfonylurea (SU) agent or insulin use [6]. Furthermore, it was reported that hypoglycemia decreases QOL of elderly individuals [7], due to decreased cognitive function $[8,9]$, exacerbation of depression symptoms [10], and increased risk of bone fracture accompanying falls [11]. Moreover, decreased QOL in elderly individuals increases the risk for cerebral stroke [12].

Thus, hypoglycemia is a significant problem in the treatment of elderly patients with diabetes; however, related details remain unclear. Therefore, to better elucidate the state of elderly T2DM patients, patient's pathophysiology, treatment, and hypoglycemia status in Japan, the Japan Physicians Association conducted the "Fact-finding Survey on Elderly Type 2
Diabetes Patients Undergoing Outpatient Treatment," a largescale questionnaire survey of physicians and their outpatients at age of 65 years or older with T2DM receiving treatment at medical institutions all over Japan, and included members of the Japan Physicians Association. Based on the results of the study, the present report focused on the feature of hypoglycemia in elderly patients with T2DM in Japan.

\section{Methods}

The "Fact-finding Survey on Elderly Type 2 Diabetes Patients Undergoing Outpatient Treatment" targeted T2DM patients aging $\geq 65$ years and receiving treatment as an outpatient in 2011. In this study, to prevent bias related to regional localities and treatment policies of particular physicians, the target number of enrolled patients was set as 10,000. Then data were collected through the cooperation of nationwide medical institutions (mainly clinics) where the internal review board was carried out at each institution, and written informed consent 
Table 1. Clinical Characteristics of Elderly T2DM Patients

\begin{tabular}{|c|c|c|}
\hline & Mean \pm SD & $\mathbf{N}$ \\
\hline Registration patients, $\mathrm{n}$ & & 15,892 \\
\hline Mean age, years & $74.2 \pm 6.3$ & 15,892 \\
\hline Gender, females (\%) & $7,516(47.5)$ & 15,835 \\
\hline Mean height, cm & $157.2 \pm 9.2$ & 14,886 \\
\hline Mean weight, kg & $59.8 \pm 11.1$ & 15,057 \\
\hline Mean BMI & $24.1 \pm 3.7$ & 14,681 \\
\hline \multicolumn{3}{|l|}{ Diabetes } \\
\hline Mean disease duration, years & $12.8 \pm 8.9$ & \\
\hline Mean onset age, years & $61.4 \pm 10.0$ & \\
\hline Mean fasting blood sugar level, $\mathrm{mg} / \mathrm{dL}$ & $128.7 \pm 34.1$ & 3,562 \\
\hline Mean HbA1c, \% (NGSP) & $7.0 \pm 1.0$ & 15,426 \\
\hline Diabetic retinopathy, n (\%) & $2,424(16.0)$ & 15,114 \\
\hline Diabetic nephropathy, n (\%) & $3,995(26.3)$ & 15,206 \\
\hline Diabetic neuropathy, n (\%) & $3,091(20.5)$ & 15,064 \\
\hline \multicolumn{3}{|l|}{ Medical history } \\
\hline Cardiovascular disease, $\mathrm{n}(\%)$ & $2,278(15.9)$ & \\
\hline Cerebrovascular disease, $\mathrm{n}(\%)$ & $1,501(10.5)$ & \\
\hline
\end{tabular}

was obtained from each participant. All data identifiable to each participant are not disclosed. Specialized survey form was distributed to physicians, who filled out items regarding the targeted patients. Survey items included patient age, disease duration, HbA1c, blood glucose levels, presence of diabetes complications, type of medication currently prescribed for diabetes, and whether the targeted patient had hypoglycemia in the last 1 month or 1 year. Hypoglycemia did not necessarily require measurement of blood glucose levels, but rather was based on the physician's judgment while considering the medical interview results. A questionnaire was simultaneously administered to all patients with the physicians' survey. The questionnaires for patients were as follows: 1) the presence or absence of symptoms in the previous month (15 items focusing on symptoms suggestive of hypoglycemia and 13 items pertaining to geriatric syndrome). These survey items were chosen in reference to Joslin's Diabetes Mellitus, 14th edition [13]; 2) knowledge regarding hypoglycemia (Do you know what hypoglycemia is?); 3) knowledge regarding hypoglycemia, such as countermeasures for hypoglycemia, symptoms, causes, and susceptible periods in a day; 4) whether the patient had experienced symptoms suspected as hypoglycemia in the last 1 month; 5) whether the patient had experienced symptoms suspected as hypoglycemia in the last 1 year; and 6) whether the patient carried glucose or an equivalent item as a countermeasure for hypoglycemia. In statistical analyses, the Chi-square test was used to compare frequencies (e.g., yes or no) between two groups, whereas the Cochran-Armitage trend test was performed for three groups or more, and logistic regression analysis was applied to identify the association between hypoglycemia and symptoms experienced by patients, and between hypoglycemia and medications (Fig. 1).

\section{Results}

Responses were received from 15,892 T2DM patients (male $52.5 \%$, female $47.5 \%$ ) aging $74.2 \pm 6.3$ years (range, $65-100$ years), with duration of diabetes of $12.8 \pm 8.9$ years, HbA1c of $7.0 \pm 1.0 \%$, and body mass index (BMI) of $24.1 \pm 3.7 \mathrm{~kg} /$ $\mathrm{m}^{2}$. Diabetes-related complications in $16.0 \%$ patients were retinopathy, in $26.3 \%$ were nephropathy (stage 2 or higher), and in $20.5 \%$ were neuropathy. In addition, $15.9 \%$ and $10.5 \%$ patients had a history of cardiovascular disturbance and cer- (a)

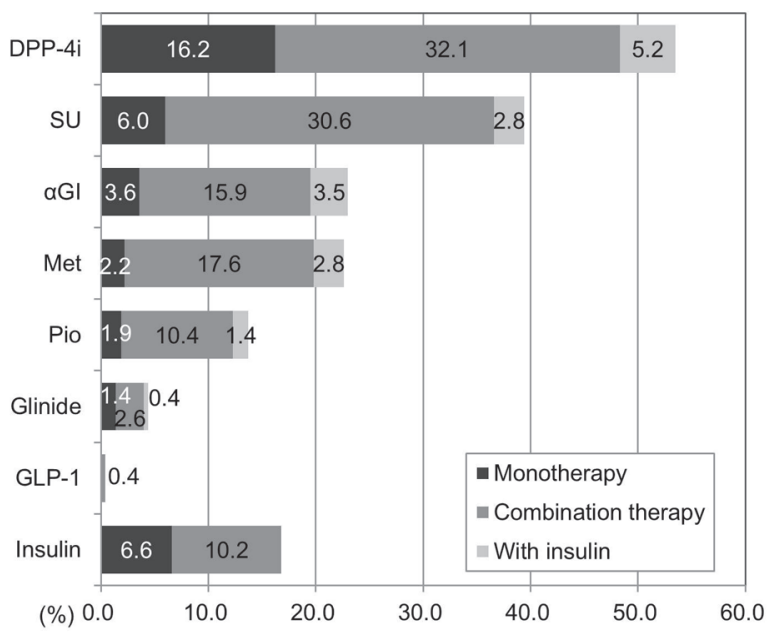

(b)

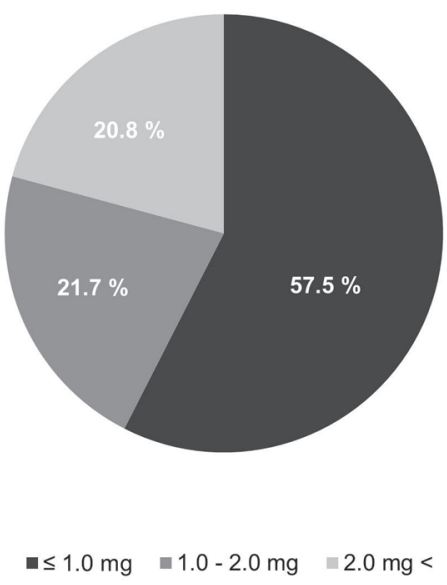

Figure 2. Trends in antidiabetic drug prescriptions. (a) Proportions of patients who were taking any one of eight drugs ( $\mathrm{n}=$ 15,855). Met: metformin; Pio: pioglitazone. (b) Patient SU dosage/day $(n=5,825)$. Conversion rate: glimepiride $1 \mathrm{mg}=$ gliclazide $40 \mathrm{mg}$ = glibenclamide $0.625 \mathrm{mg}$. 


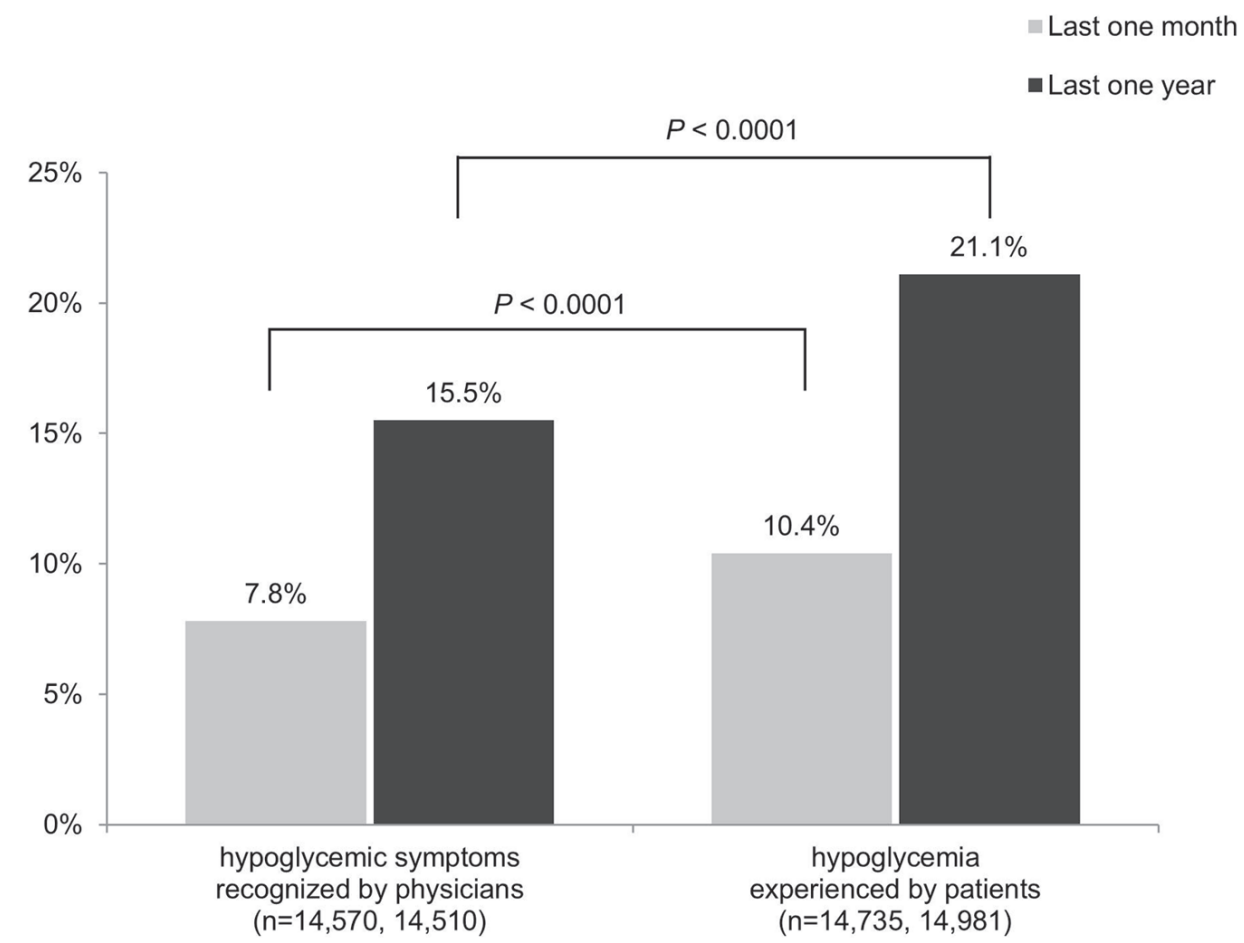

Figure 3. State of hypoglycemia.
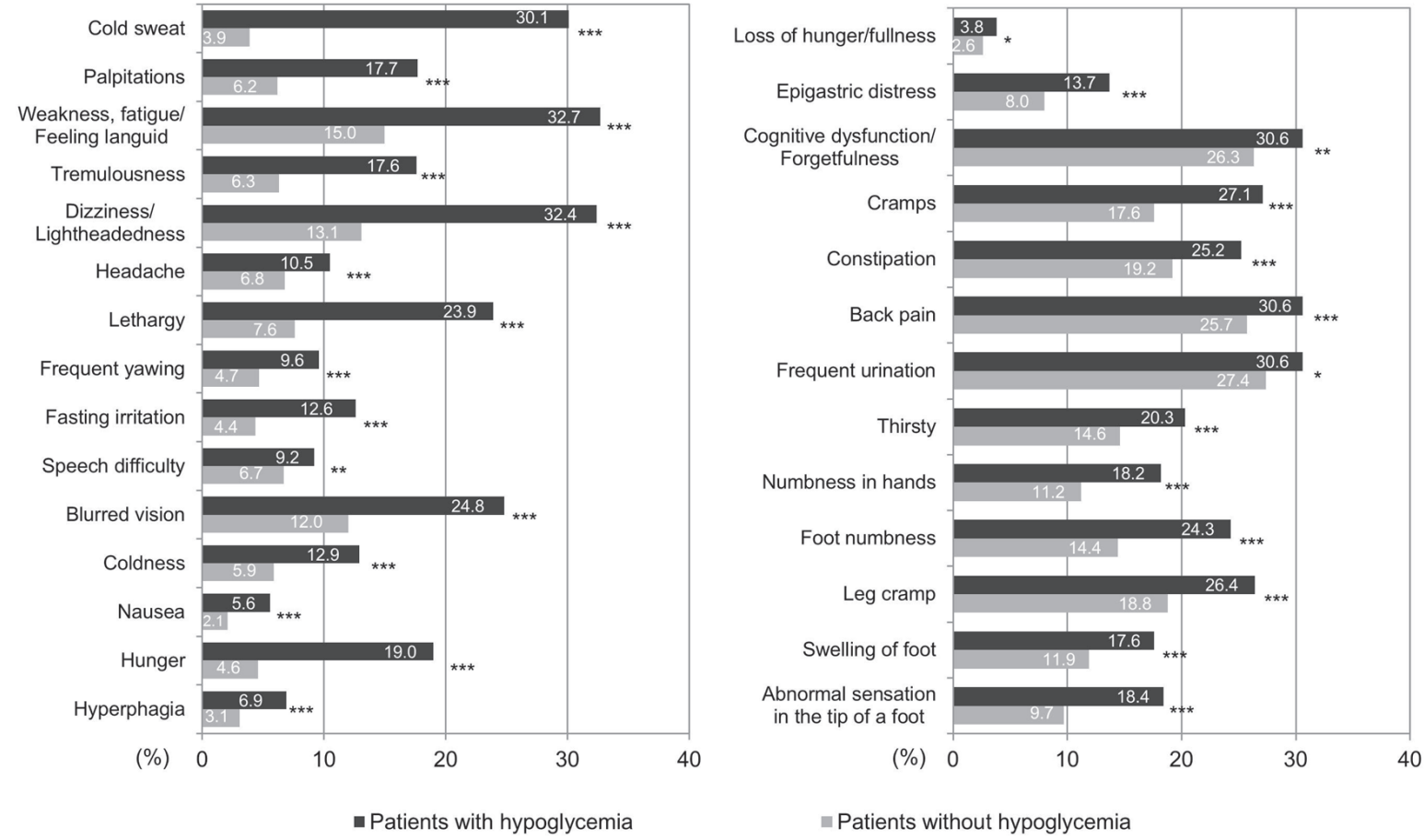

Figure 4. Hypoglycemia in patients with the 28 symptoms. Patients with hypoglycemia commonly presented with weakness, fatigue/feeling languid, dizziness/lightheadedness, cognitive dysfunction/forgetfulness, back pain, and frequent urination, and cold sweats. ${ }^{*} \mathrm{P}<0.05,{ }^{* *} \mathrm{P}<0.01,{ }^{* * *} \mathrm{P}<0.001$. 


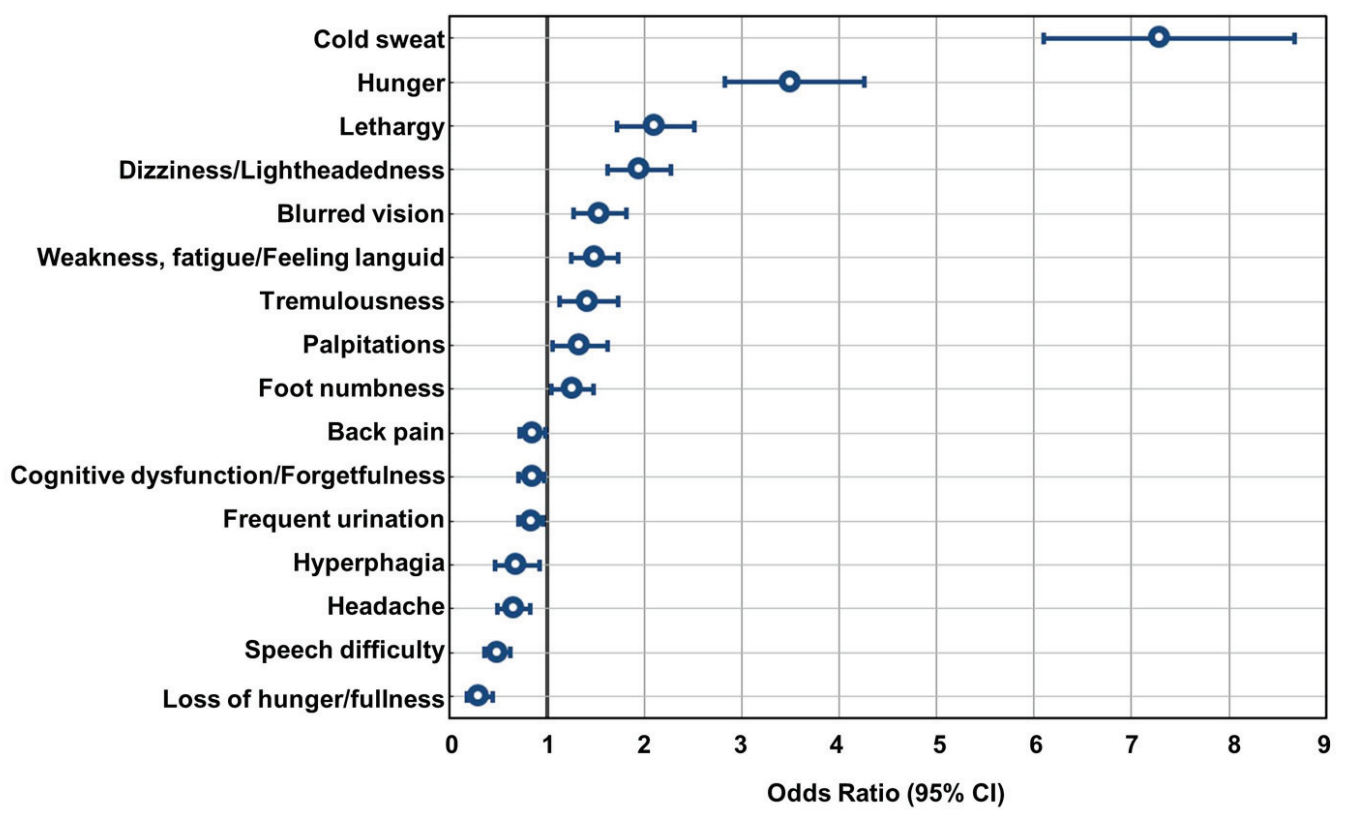

Figure 5. Associations between hypoglycemia and the symptoms. Multiple logistic regression analysis of the incidence of hypoglycemia by doctors' records in the last 1 month and the 28 self-reported symptoms by patients is shown above. "Cold sweat" and "hunger" indicated a strong positive relationship with the incidence of hypoglycemia. On the other hand, we found some symptoms like "loss of hunger/fullness" showed negative association with the incidence of hypoglycemia.

ebrovascular disease, respectively (Table 1).

\section{Prescriptions of oral hypoglycemic agents (OHAs) for el- derly diabetes patients}

Figure 2a shows the frequency of prescriptions for medications. The highest prescription rate for OHAs was for dipeptidyl peptidase-4 (DPP-4) inhibitors, which were prescribed to over $50 \%$ of patients (monotherapy, $16.2 \%$; combined therapy, $37.3 \%$ ). The second highest prescription rate was for SU agents (monotherapy, 6.0\%; combined therapy, 33.4\%), followed by alpha-glucosidase inhibitors ( $\alpha$-GIs; monotherapy, $3.6 \%$; combined therapy, 19.4\%), metformin (monotherapy, 2.2\%; combined therapy, $20.4 \%$ ), pioglitazone (monotherapy, $1.9 \%$; combined therapy, $11.8 \%$ ), and glinide agents which were less commonly prescribed (monotherapy, $1.4 \%$; combined therapy, $3 \%$ ). Regarding the type of SU agents used, $80.7 \%$ of prescriptions were for glimepiride, $8.8 \%$ for gliclazide, and $10.5 \%$ for glibenclamide (not shown in the figure). A 1-day dosage was converted as follows: $1 \mathrm{mg}$ of glimepiride $=40 \mathrm{mg}$ of gliclazide $=0.625 \mathrm{mg}$ of glibenclamide.

Using this formula to convert results for glimepiride, the most commonly administered SU agent, we observed that 1.0 $\mathrm{mg}$ /day or less was administered to $57.5 \%$ patients, $1.0-2.0$ $\mathrm{mg}$ /day was administered to $21.7 \%$, and $>2.0 \mathrm{mg} /$ day was administered to $20.8 \%$. With the mean dosage of $1.94 \mathrm{mg} / \mathrm{day}$, the amounts of SU prescription were relatively low (Fig. 2b).

Regarding injection therapy, GLP-1 agonists were administered to $0.4 \%$ patients, whereas insulin therapy was adminis- tered to $16.8 \%$ of patients (Fig. 2a).

\section{State of hypoglycemia}

As shown in Figure 3, the overall frequency of patients' hypoglycemia that physicians recognized in the last 1 month was $7.8 \%$. This significantly differed from patient questionnaire results, in which $10.4 \%$ of patients had experienced hypoglycemia in the last 1 month $(\mathrm{P}<0.0001)$. The rates of recognition for hypoglycemia over the last 1 year also significantly differed: $15.5 \%$ and $21.1 \%$ for physicians and patients, respectively $(\mathrm{P}<0.0001)$. When insulin treatment was excluded, which is not shown in the figure, the frequencies of hypoglycemia for the last 1 month and the last 1 year were $3.8 \%$ and $8.8 \%$, respectively, according to the physicians, and $6.1 \%$ and $13.8 \%$, respectively, according to the patients.

Regarding symptoms experienced subjectively by patients in the last 1 month, Figure 4 shows results with patients divided into groups according to whether they answered that they did or did not experience hypoglycemia. The most common symptom, "weakness, fatigue/feeling languid," was noted in $32.7 \%$ patients in the hypoglycemia group, whereas it was noted only in $15.0 \%$ patients in the non-hypoglycemia group. Furthermore, in the hypoglycemia and non-hypoglycemia groups, "dizziness/lightheadedness" was experienced by $32.4 \%$ vs. $13.1 \%$, respectively, followed by "cognitive dysfunction/forgetfulness" by $30.6 \%$ vs. $26.3 \%$, "back pain" by $30.6 \%$ vs. $25.7 \%$, "frequent urination" by $30.6 \%$ vs. $27.4 \%$, and "cold sweat" by $30.1 \%$ vs. $3.9 \%$, respectively. Thus, neuroglyco- 
Table 2. Hypoglycemia Symptoms and Age (Total $N=1,142$ )

\begin{tabular}{llllll}
\hline & $\mathbf{6 5 - 7 0}(\mathbf{n}=\mathbf{3 1 2})$ & $\mathbf{7 0 - 7 5}(\mathbf{n = 3 2 7})$ & $\mathbf{7 5 - 8 0}(\mathbf{n}=\mathbf{2 9 8})$ & $\mathbf{2 8 0}(\mathbf{n}=\mathbf{2 0 5})$ & P value* \\
\hline Cold sweat & 32.1 & 31.2 & 31.2 & 24.1 & 0.09 \\
Dizziness/lightheadedness & 27.6 & 31.5 & 34.6 & 41.4 & 0.01 \\
Weakness, fatigue/feeling languid & 29.5 & 29.1 & 37.2 & 33.8 & 0.02 \\
Blurred vision & 22.4 & 25.4 & 26.8 & 26.2 & 0.46 \\
Lethargy & 24.7 & 20.2 & 21.8 & 31.7 & 0.13 \\
\hline
\end{tabular}

${ }^{*}$ Cochran-Armitage trend test.

penic symptoms such as "weakness, fatigue/feeling languid," "dizziness/lightheadedness," and "cognitive dysfunction/forgetfulness" were most common, whereas among the six most experienced symptoms, "cold sweat" was the only autonomic symptom noted.

Many of the above symptoms were also noted by patients without hypoglycemia. We also performed multivariate analysis to assess the relationship between the absence or presence of hypoglycemia and symptoms of hypoglycemia (Fig. 5). Results indicated that "cold sweat" had the highest odds ratio (OR) of 7.4, followed by "hunger," "lethargy," "dizziness/lightheadedness," "blurred vision," "weakness, fatigue/ feeling languid," "tremulousness," "palpitations," and "foot numbness." These symptoms appear to have some correlation with the presence or absence of hypoglycemia (Fig. 5). For "cold sweat" and "hunger," both parasympathetic nervous system symptoms, the strongest and second strongest correlations were observed, respectively. These were followed by symptoms originated from glucose deficiency in the central nervous system, and unidentified complaints. Then, "palpitations" and "tremulousness," which are symptoms originating in the sympathetic nervous system, had the lowest incidences. The ORs were low for "cognitive dysfunction/forgetfulness"

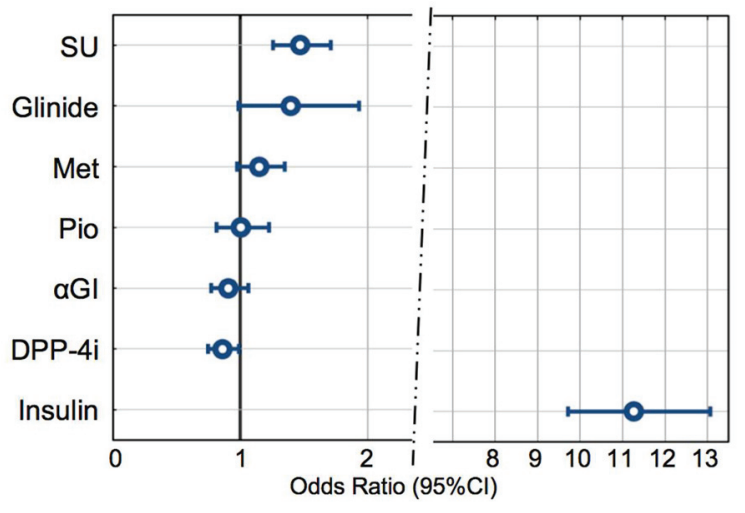

Figure 6. Associations between hypoglycemia and drugs. A multiple logistic regression analysis was performed between the incidence of hypoglycemia in the last 1 month, as assessed by doctors, as the dependent variable to analyze the associations with drug use. SU: significantly high OR (odds ratio). Glinide, Met, Pio, aGI: not significant. DPP-4i: the lowest OR $(<1)$. Insulin: prominently high OR. GLP-1 and other drugs were excluded from this analysis due to the lower number of patients using the drugs. and "headache," which are categorized as central nervous system hypoglycemic symptoms. In our examination whether there were any characteristics of the symptoms observed when groups were stratified by age, such as the oldest old, symptoms such as "dizziness/lightheadedness" and "weakness, fatigue/ feeling languid" was significantly more common as the elderly patients aged. However, "cold sweating" tended to decrease in those aging $\geq 80$ years although it was not significant (Table 2).

\section{Factors associated with the incidence of hypoglycemia}

We investigated the relationship between the use of OHAs and incidence of hypoglycemia. In the 1,140 patients who were recognized by a physician to have experienced hypoglycemia in the last 1 month and were using diabetes medication as a monotherapy, the highest incidence of hypoglycemia was observed in the patients undergoing insulin monotherapy $(32.7 \%)$. With regard to monotherapy with OHAs, incidence of hypoglycemia was high in the patients using drugs to promote insulin secretion as $4 \%$ for SU agents and 3.8\% for glinide agents, followed by $3.5 \%$ for pioglitazone, whereas $\leq 2 \%$ in patients using metformin, DPP-4 inhibitors, or $\alpha$-GIs. No significant differences were detected in the incidence of hypoglycemia between the patients with glinide agents and with other OHAs; however, the incidence of hypoglycemia was significantly higher in patients using SU agents than those using metformin, DPP-4 inhibitors, or $\alpha$-GIs ( $\mathrm{P}=0.0257, \mathrm{P}=0.0001$, and $\mathrm{P}=0.0301$, respectively).

We also performed multivariate analysis to assess the relationship between the use of antidiabetic agents, including combined therapy, and incidence of hypoglycemia. As shown in Figure 6, the incidence of hypoglycemia was highest in patients receiving insulin therapy (OR 11.4). In the OHAs, SU agents exhibited a significantly higher incidence of hypoglycemia (OR 1.47), demonstrating that hypoglycemia was high in patients using SU agents. The OR for glinide agents was relatively high as 1.29 but did not show statistical significance. No statistically significant differences were noted for metformin, pioglitazone, or $\alpha$-GIs. DPP-4 inhibitors showed OR less than 1 as 0.86 , which was statistically significant; this suggested that incidence of hypoglycemia was low in patients using DPP-4 inhibitors.

The relationship between the number of patients who had experienced hypoglycemic symptoms recognized by a physician and $\mathrm{HbA1c}$, BMI, duration of diabetes, and three major 

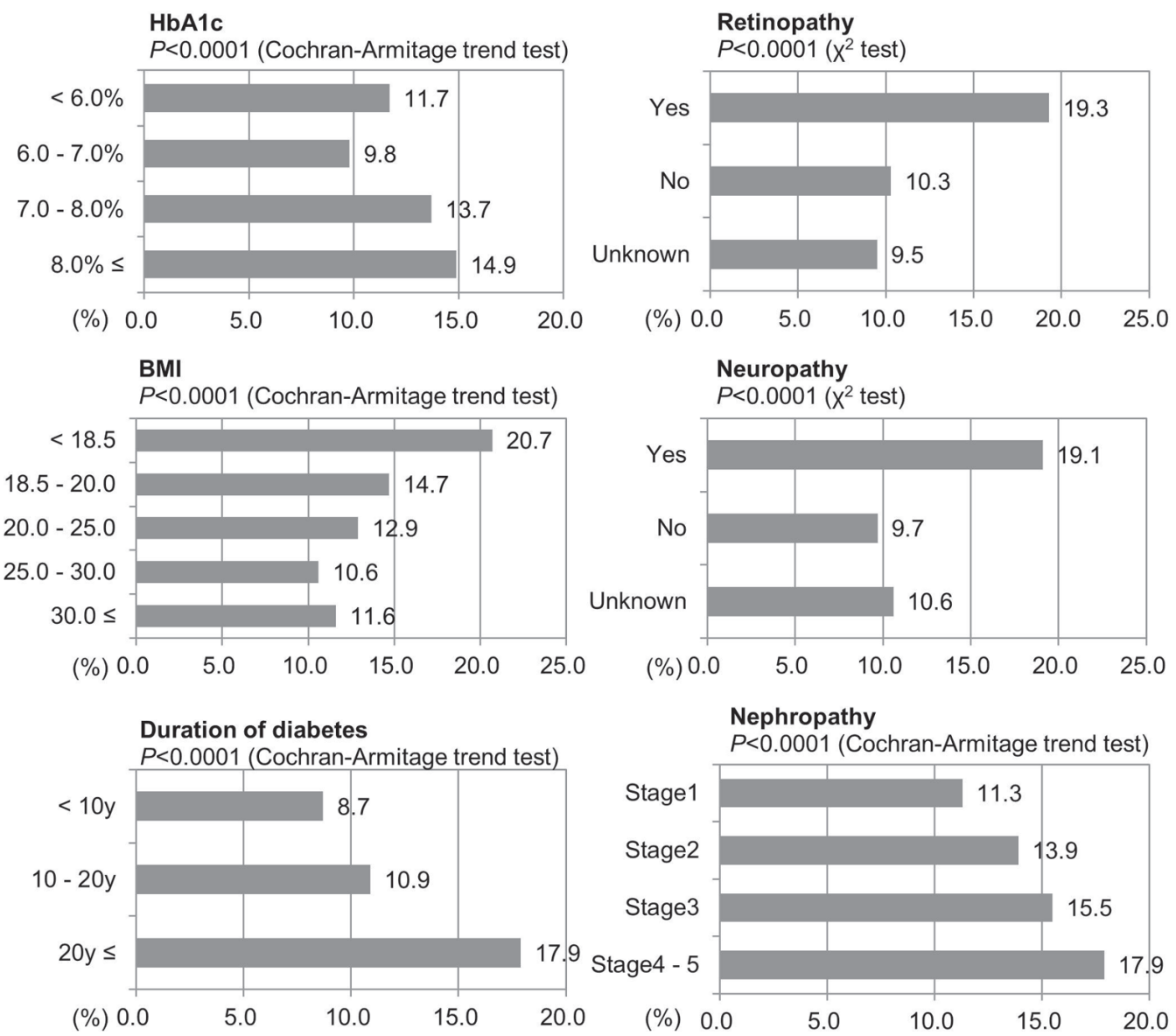

Figure 7. Hypoglycemia in the patients using SU or insulin. Percent of incidence of hypoglycemia based on doctors' records.

complications of diabetes were investigated (Fig. 7). Results for $\mathrm{HbAlc}$ indicated that patients with poorer blood glucose control, lower BMI and longer disease duration tended to be more likely to have experienced hypoglycemia $(\mathrm{P}<0.0001)$.

Patients with complications of retinopathy or neuropathy had a significantly higher incidence of hypoglycemia than those without such complications (both, $\mathrm{P}<0.0001$ ). Similarly, patients with nephropathy were significantly more likely to have experienced hypoglycemia than those without nephropathy (stage 1 as no nephropathy). As the degree of nephropathy worsened, this trend intensified $(\mathrm{P}<0.0001)$.

\section{Patients' knowledge regarding hypoglycemia}

Overall, $71.5 \%$ patients responded positively to the question, "Do you know what hypoglycemia is?": 91.4\% patients receiving insulin therapy, $75.8 \%$ using SU agents, and $64.3 \%$ not using insulin or SU agents (Fig. 8). Patients who had used SU agents or insulin had significantly more knowledge of hypoglycemia than those being treated with other drugs $(\mathrm{P}<0.001)$. In the question "knowledge of countermeasures for hypoglycemia", the majority of patients, with insulin therapy (both monotherapy and combined therapy), with SU agents (both monotherapy and combined therapy), and those not using insulin or SU agents, had high knowledge of countermeasures for hypoglycemia $(83.9 \%, 78.8 \%$ and $72.9 \%$, respectively). Although most of patients in the study had some knowledge of countermeasures, more insulin users compared to SU users are aware of the countermeasures. Similarly, 54.0\% patients using insulin therapy, $44.0 \%$ using SU agents, and 37.4\% not using insulin or SU agents had knowledge of symptoms but experienced by hypoglycemia. Unfortunately only $36.5 \%$ patients receiving insulin therapy, $25.2 \%$ using SU agents, and $21.7 \%$ of those not using insulin or SU agents had knowledge of the causes of hypoglycemia. Finally, merely $29.4 \%$ patients receiving insulin therapy, $19.7 \%$ using SU agents, and $14.7 \%$ not using insulin or SU agents had low recognition about times when hypoglycemia was likely to occur.

Regardless of the high level of knowledge regarding hypoglycemia, only $63 \%$ patients receiving insulin therapy and $31 \%$ using SU agents responded that they always carried glucose or a similar medication with them; this fact confirmed that many patients had knowledge of hypoglycemia but were not executing countermeasures to prevent its onset. Furthermore, we observed that the number of the patients who always car- 


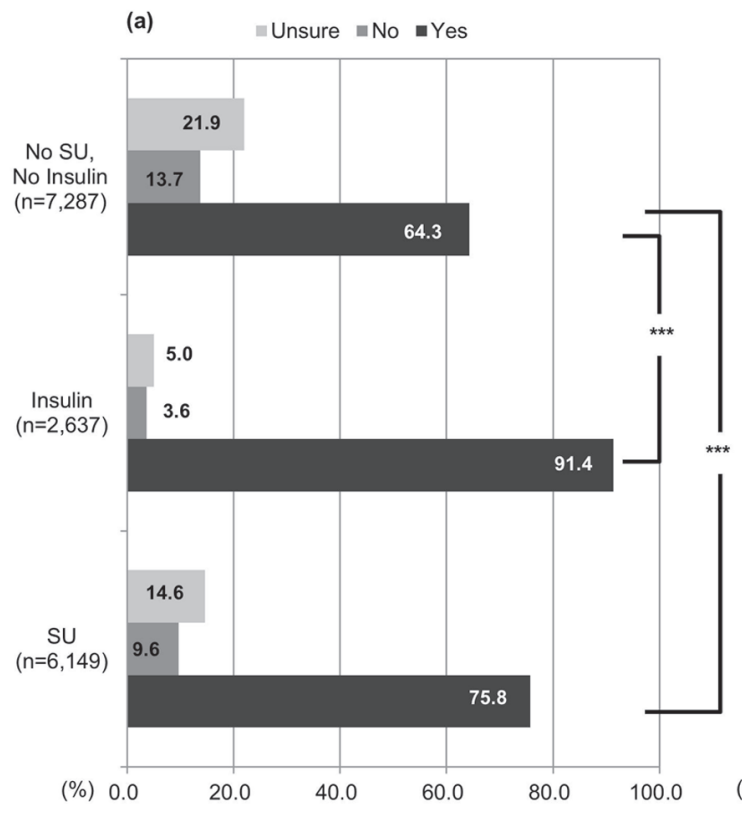

(b) $\quad \square$ Time $\square$ Cause $\square$ Symptom $\quad$ Treatment

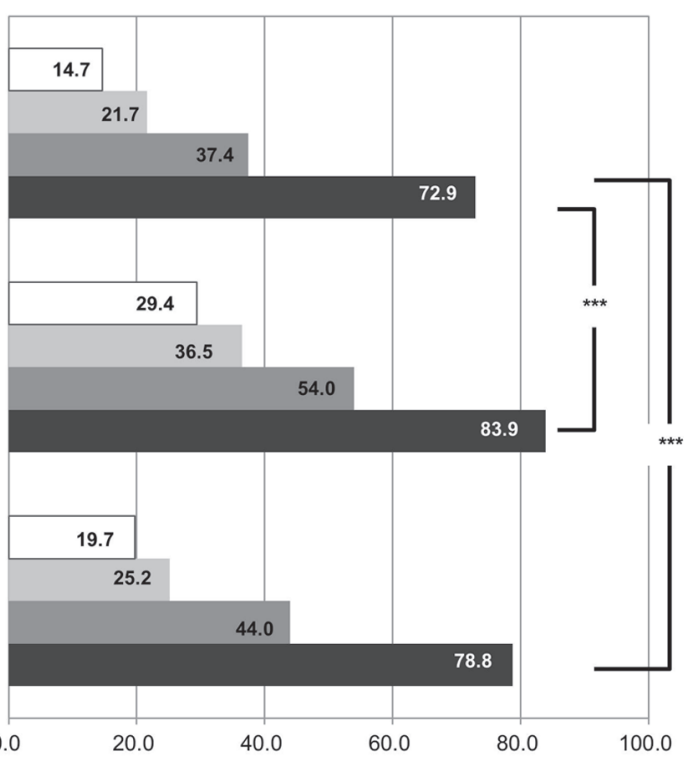

Figure 8. Knowledge of hypoglycemia. (a) "Are you aware of hypoglycemia?" Hypoglycemia awareness in elderly patients who were taking SU, insulin, or neither. (b) "What do you know about hypoglycemia?" Patients who chose "yes" to the first question answered this question. ${ }^{*} \mathrm{P}<0.05,{ }^{* *} \mathrm{P}<0.01,{ }^{* \star *} \mathrm{P}<0.001$.

ried glucose with them decreased with age; only $49 \%$ patients receiving insulin therapy and $21.7 \%$ using SU agents carried glucose in the patients aging $\geq 85$ years.

\section{Discussion}

In recent years, it has become clear that, when treating diabetes mellitus, hypoglycemia - particularly severe hypoglycemia causes worsening of the incidence and prognosis of cerebrovascular events [14], and knowing how to prevent hypoglycemia and control diabetes mellitus has gained great attention. In reports dealing with severe hypoglycemia, background factors included advanced age [15], decreased renal function [16], SU agents [17], and insulin use [18]. To prevent such severe hypoglycemia occurring, hypoglycemia should not be overlooked in elderly patients with diabetes during routine treatment, and it is important that proper measures should be taken. The conditions of elderly T2DM patients receiving treatment in Japan, and symptoms and frequency of hypoglycemia remain unclear. Hence, we investigated hypoglycemia based on the results of a survey conducted on the general internists treating patients and the patients commuting to their medical institutions.

\section{Definition of hypoglycemia in elderly T2DM patients and its relationship with the incidence}

In the present study, the incidence of hypoglycemia diagnosed by physicians was $7.8 \%$ in the last 1 month and $15.5 \%$ in the last 1 year. This frequency is the aggregate of hypoglycemia incidences observed subjectively by primary physicians and that reported by patients; there was no measurement of blood glucose levels, such as the self-monitoring blood glucose levels. There were no strict specifications regarding the rationale for diagnosing the occurrence of hypoglycemia in patients by primary physicians; it was judged based on the subjective opinion of the primary physician. The definition of severity for hypoglycemia is also vague because it was not classified into mild and severe. However, the incidence of hypoglycemia reported in this study was similar to that found in a registry study recently reported in Germany [19]. Targets of the registry studies were patients with T2DM aging $\geq 40$ years and using 1 - 2 types of OHAs. The incidence of hypoglycemia during 1 year was $10.5 \%$, as obtained from responses reported by patients [19]. UKPDS73 [18] also showed slightly lower incidence at $11 \%$ for 1 year; however, other prospective 1-year registry studies showed an incidence of $14.1 \%$ [20]. The authors in the study discussed that it estimated as a lower rate of hypoglycemia in retrospective investigations but the incidence rate was similar to that observed in this study.

Furthermore, the symptoms experienced by most patients during the present study fairly correspond to the symptoms of hypoglycemia in Joslin's Diabetes Mellitus (14th edition). Therefore, we believe it is sufficiently useful for diagnosing hypoglycemia in elderly T2DM patients.

Notably, irrespective of whether the time period was 1 month or 1 year, there were significant differences in awareness of hypoglycemia between physicians and patients. This suggested the presence of "hidden hypoglycemia that physicians did not detect." According to Gotoh et al, that is an investigation of patients with diabetes being treated with SU agents 
or insulin therapy, $49 \%$ of patients in whom hypoglycemia occurred did not report the incidence to their primary physician [21]. In Japan, the time to examine a single patient in clinical practice is short and approximately half of these are completed under $10 \mathrm{~min}$ [22]. It was also reported that impact of diabetes mellitus on the patients' lifestyles has not been sufficiently asked [3]; therefore, we believe that it results in differences in the understanding of hypoglycemia between physicians and patients. We suggest that it is important to confirm the presence of hypoglycemia by focusing on asking regarding definite symptoms when examining patients who are susceptible to hypoglycemia during each examination.

In addition, it must be noted that elderly patients with diabetes may describe a sign or symptom of hypoglycemia as general tiredness during physical examination. Even during the present study, in addition to symptoms such as "back pain" and "frequent urination," which were believed to be due to geriatric syndrome, symptoms of general malaise such as "cramps", "numbness in the legs", and "swelling" and "easy to get foot cramps" appeared frequently. A relationship between diabetes mellitus/hypoglycemia and symptoms such as "cramps" has been reported [23, 24]; therefore when asking about hypoglycemia during routine examination, physicians should inquire patients regarding not only the known conventional symptoms of hypoglycemia but also the various symptoms of general malaise shown that many patients have in this study.

Normally, the most frequent symptoms of hypoglycemia are symptoms caused by autonomic nervous system, such as "sweating" and "palpitations," and the most common central nervous system symptoms, such as "confusion," "lightheadedness," and "malaise" [25]. However, in patients aging $\geq 70$ years, central nervous system symptoms of mild headache and unsteadiness are reported to be common [26]; the present study also showed that the OR of the symptoms originating from the sympathetic nervous system was low, and the parasympathetic symptoms were ranked higher in the autonomic symptoms.

In addition, the multivariate analysis for symptoms experienced by patients in 1 month and symptoms of hypoglycemia, OR of central nervous system symptoms of hypoglycemia listed in Joslin's Diabetes Mellitus (14th edition), i.e., "forgetfulness" and "headache," were low. We consider that this is because the patients had poor knowledge that the symptoms such as "forgetfulness" and "headache" are hypoglycemic symptoms. Therefore these can be "hidden hypoglycemia because of the patients' lack of knowledge." It is important that the physicians instruct their patients to take glucose immediately when an atypical hypoglycemic symptom appears and confirm whether a symptom is improved.

The incidence of hypoglycemia does not increase when $\mathrm{HbA1c}$ decreases, rather hypoglycemic risks increase when HbAlc values rise. The incidence of hypoglycemia was investigated in 7,855 patients using SU agents or insulin; the incidence of hypoglycemia increased in both the groups with low and high $\mathrm{HbAlc}$. Other studies have also reported that the risk of hypoglycemia increases in patients with poor glycemic control $[27,28]$, and notably, hypoglycemia needs to be taken into account in elderly Japanese T2DM patients who have high HbA1c values. In addition, it is known that the risk of hypoglycemia is typically higher in patients with a long disease du- ration or those with diabetic complications, including young patients [29]; similar results are also found in the present study. We believe closer attention needs to be paid to hypoglycemia in elderly patients with lower BMI and poor glycemic control.

\section{Relationship between hypoglycemia and antidiabetic drugs}

Similar to previous studies [30, 31], insulin was associated with an exceptionally high incidence of hypoglycemia, and the incidence was high in patients using SU agents or glinides as OHA monotherapy. The risk of hypoglycemia for patients using DPP-4 inhibitors was the smallest. Similar trends were found in analysis with combined therapies, a strong association with not only insulin but also SU agents and glinides, a low risk of hypoglycemia when using DPP-4 inhibitors. This shows that DPP-4 inhibitors are effective at preventing hypoglycemia and have a lower incidence of adverse events than other OHAs [32]. In addition to these safety aspects, there is abundant useful evidence for their use in elderly T2DM patients as they improve symptoms of dementia [33] and bone metabolism [34]. It is why DPP-4 inhibitors are commonly used as first-line treatment.

Although SU agents have a high risk of hypoglycemia, $34 \%$ of patients even with $\mathrm{HbAlc} \leq 7 \%$ used SU agents in the present study, indicating that these patients are with a latent risk of hypoglycemia. The average dose of SU agents is comparatively low in the present study; however, a report has suggested that even a small amount of SU agents could put elderly patients at risk of experiencing hypoglycemia [35]. For this reason, great attention is required when managing hypoglycemia, even at low doses. Furthermore, even DPP-4 inhibitors, its use combined with SU agents could cause severe hypoglycemia, and also reported that the incidence frequency of severe hypoglycemia changes based on the type of SU used in the combination [36]. Among the SU agents, compared with glibenclamide or glimepiride which have a high affinity for SU receptor on the pancreatic B cells, gliclazide which has a long half-life and a weak affinity for the receptors has been reported to show low frequencies of mild and severe cases of hypoglycemia; however, this tendency was not observed in the present study. This suggested the requirement for caution when using any kinds of SU agents in the elderly. In practice, as shown in other epidemiological studies, the frequency of SU agents use decreases with time [37]; however, the use of SU agents is frequently used concomitantly in Japan, and attention is thus required on the effects of any combination use.

\section{Knowledge of hypoglycemia}

We found that patients using insulin and SU agents have a higher level of knowledge of hypoglycemia than patients using other drugs. This is considered to be because many patients have experienced hypoglycemia due to their trends that these drugs readily cause hypoglycemia, and have been frightened by the experience [38]. However, there is an investigative report stating that patients do not proactively notify their physicians when hypoglycemia occurs [21]; another report has 
stated that primary physicians have insufficient knowledge regarding patients' hypoglycemia [39]. Particularly among routine medical treatments, it is important to ask them regarding hypoglycemia during each visit, when patients are using insulin and SU agents.

Even among the patients who answered that they "had not experience hypoglycemia," many patients presented with hypoglycemic-like symptoms, and it is likely that the number of patients who have actually experienced hypoglycemia is higher than the number. This also suggested the presence of "hidden hypoglycemia that patients were not aware." Normally, glucagon and adrenaline, the so-called anti-insulin hormones, are secreted when the blood glucose levels decrease and the autonomic symptoms occurring due to secretion of adrenaline may serve as a warning sign for hypoglycemia. At this point, prevention of severe hypoglycemia is possible if it is noticed and glucose is ingested. If it is not done promptly, the blood glucose levels will decrease further until central nervous system symptoms, such as decreased cognition, appear and it becomes difficult to treat the hypoglycemia, and the possibility of becoming severe hypoglycemia is high. In elderly patients, in particular, central nervous system symptoms such as slurred speech and incoordination appear more readily than autonomic symptoms [26], and the blood glucose threshold for appearance of autonomic symptoms is lower compared with younger patients. On the other hand, the threshold for blood glucose level for the appearance of central nervous system symptoms is higher in elderly patients [40]; therefore, this is one factor that delays the appropriate management of hypoglycemia. With advanced age, the ability to recognize hypoglycemia weakens [4], and there are some cases where the patients themselves are unaware of hypoglycemia. Acquiring knowledge regarding hypoglycemia reinforces subjective perception of hypoglycemia [41], and it is important to raise awareness of hypoglycemia to prevent serious situations.

In the present study, the degree of knowledge regarding hypoglycemia was comparatively good overall, but when we examined the details, frequency of patients who knew "what time periods were associated with the appearance of hypoglycemia" was low. The response to questions indicating preparedness for hypoglycemia, such as "Do you have glucose on you?" was lower than expected in patients using SU agents, and the tendency was more common as the patients aged. Thus, during routine examination, the physicians should regularly provide guidance regarding how to manage hypoglycemia, and it is also important to actually hand glucose to patients. It may be possible that elderly patients cannot understand the types of diabetic medication that was prescribed to them, although a survey item for it was not included in the present study. In any case, the treatment should be explained to patient themselves and a key member of the family, and basic guidance should be provided regarding the methods of managing hypoglycemia; this guidance should be continuously repeated in the future.

\section{Conclusion}

The results of the present study suggested the presence of two types of "hidden hypoglycemia", namely: "hidden hypogly- cemia that physicians did not detect" and "hidden hypoglycemia that patients were not aware." Compared with younger patients, elderly hypoglycemic patients more commonly have atypical symptoms of hypoglycemia, and the hypoglycemia is not noticed in the first place. These conditions also promote the development of severe hypoglycemia [4]. In addition, consistent hypoglycemic symptoms do not necessarily appear in the same patient [42]. Further investigation of the relationship between hypoglycemia and various symptoms in elderly T2DM patients, including unidentified complaints, should be encouraged. In addition to establishing a highly precise tool enabling easy diagnosis of the appearance of hypoglycemia in patients by the family physician, the patient should also be proactively inquired regarding whether they have such symptoms. It is vital that physicians strive to prevent overlooking hypoglycemic symptoms. Furthermore, instructions should be provided to the patients regarding glucose intake when these symptoms appear. The patients' own degree of understanding and readiness for hypoglycemia must be important for its prevention.

\section{Acknowledgement}

We thank the Japan Physicians Association and the 1,086 institutes in Japan for their cooperation with this study. This study was supported by Ono Pharmaceutical Co., Ltd, so we thank them also to make the important facts visible to the public.

\section{Funding}

The original research was conducted by the Japan Physicians Association, and was supported by Ono Pharmaceutical Co., Ltd.

\section{Conflict of Interest}

No conflict of interests is involved in this study.

\section{References}

1. IDF diabetes atlas sixth edition website. International Diabetes Federation. http://www.idf.org/sites/default/files/ Atlas-poster-2014_EN.pdf. Accessed May 13, 2015.

2. Ministry of Health, Labour and Welfare. http://www. mhlw.go.jp/toukei/list/dl/81-1a2.pdf. Accessed July 30, 2015.

3. Nicolucci A, Kovacs Burns K, Holt RI, Comaschi M, Hermanns N, Ishii H, Kokoszka A, et al. Diabetes Attitudes, Wishes and Needs second study (DAWN2): cross-national benchmarking of diabetes-related psychosocial outcomes for people with diabetes. Diabet Med. 2013;30(7):767-777.

4. Bremer JP, Jauch-Chara K, Hallschmid M, Schmid S, Schultes B. Hypoglycemia unawareness in older com- 
pared with middle-aged patients with type 2 diabetes. Diabetes Care. 2009;32(8):1513-1517.

5. Iwakura T, Sasaki S, Fujiwara Y, Matsuoka N, Ishihara T. Clinical analysis of 135 type 2 diabetes patients with severe drug-induced hypoglycemia. Diabetes. 2012;55(11):857-865.

6. Haneda M, Morikawa A. Which hypoglycaemic agents to use in type 2 diabetic subjects with CKD and how? Nephrol Dial Transplant. 2009;24(2):338-341.

7. Rombopoulos G, Hatzikou M, Latsou D, Yfantopoulos J. The prevalence of hypoglycemia and its impact on the quality of life (QoL) of type 2 diabetes mellitus patients (The HYPO Study). Hormones (Athens). 2013;12(4):550558.

8. Korf ES, White LR, Scheltens P, Launer LJ. Brain aging in very old men with type 2 diabetes: the Honolulu-Asia Aging Study. Diabetes Care. 2006;29(10):2268-2274.

9. Yaffe K, Falvey CM, Hamilton N, Harris TB, Simonsick EM, Strotmeyer ES, Shorr RI, et al. Association between hypoglycemia and dementia in a biracial cohort of older adults with diabetes mellitus. JAMA Intern Med. 2013;173(14):1300-1306.

10. Kikuchi Y, Iwase M, Fujii H, Ohkuma T, Kaizu S, Ide H, Jodai T, et al. Association of severe hypoglycemia with depressive symptoms in patients with type 2 diabetes: the Fukuoka Diabetes Registry. BMJ Open Diabetes Res Care. 2015;3(1):e000063.

11. Tilling LM, Darawil K, Britton M. Falls as a complication of diabetes mellitus in older people. J Diabetes Complications. 2006;20(3):158-162.

12. Araki A, Murotani Y, Kamimiya F, Ito H. Low well-being is an independent predictor for stroke in elderly patients with diabetes mellitus. J Am Geriatr Soc. 2004;52(2):205210.

13. Glaser B, Leibowitz G. Hypoglycemia. In: Kahn CR, Weir GC, King GL, Jacobson AM, Moses AC, Smith RJ, eds. Joslin's Diabetes Mellitus. 14th ed. Philadelphia: Lippincott, 2005:1147-1176.

14. Hsu PF, Sung SH, Cheng HM, Yeh JS, Liu WL, Chan WL, Chen $\mathrm{CH}$, et al. Association of clinical symptomatic hypoglycemia with cardiovascular events and total mortality in type 2 diabetes: a nationwide population-based study. Diabetes Care. 2013;36(4):894-900.

15. Seltzer HS. Drug-induced hypoglycemia. A review of 1418 cases. Endocrinol Metab Clin North Am. 1989;18(1):163-183.

16. Goto A, Arah OA, Goto M, Terauchi Y, Noda M. Severe hypoglycaemia and cardiovascular disease: systematic review and meta-analysis with bias analysis. BMJ. 2013;347:f4533.

17. Hou L, Zhao T, Liu Y, Zhang Y. Efficacy and safety of sitagliptin compared with sulfonylurea therapy in patients with type 2 diabetes showing inadequately controlled glycosylated hemoglobin with metformin monotherapy: A meta-analysis. Exp Ther Med. 2015;9(4):1528-1536.

18. Wright AD, Cull CA, Macleod KM, Holman RR. Hypoglycemia in Type 2 diabetic patients randomized to and maintained on monotherapy with diet, sulfonylurea, metformin, or insulin for 6 years from diagnosis: UKPDS73.
J Diabetes Complications. 2006;20(6):395-401.

19. Tschope D, Bramlage P, Binz C, Krekler M, Plate T, Deeg E, Gitt AK. Antidiabetic pharmacotherapy and anamnestic hypoglycemia in a large cohort of type 2 diabetic patients--an analysis of the DiaRegis registry. Cardiovasc Diabetol. 2011;10:66.

20. Tschope D, Bramlage P, Binz C, Krekler M, Deeg E, Gitt AK. Incidence and predictors of hypoglycaemia in type 2 diabetes - an analysis of the prospective DiaRegis registry. BMC Endocr Disord. 2012;12:23.

21. Gotoh Y, Kaku K, Ishizaki M, Asamura R, Yamamoto Y, Nakata K, et al. A consciousness investigation of low blood sugar levels in patients with glycosuria. Japanese Society of Hospital Pharmacists Journal. 2005;41(11):1437-1439.

22. Ministry of Health, Labour and Welfare. Patient's Behavior Survey 2011 (in Japanese). http://www.mhlw.go.jp/ toukei/saikin/hw/jyuryo/11/. Accessed July 30, 2015.

23. Kim DH, Yoon DM, Yoon KB. The effects of myofascial trigger point injections on nocturnal calf cramps. J Am Board Fam Med. 2015;28(1):21-27.

24. Merlino G, Fratticci L, Valente M, Del Giudice A, Noacco C, Dolso P, Cancelli I, et al. Association of restless legs syndrome in type 2 diabetes: a case-control study. Sleep. 2007;30(7):866-871.

25. Deary IJ, Hepburn DA, MacLeod KM, Frier BM. Partitioning the symptoms of hypoglycaemia using multi-sample confirmatory factor analysis. Diabetologia. 1993;36(8):771-777.

26. Jaap AJ, Jones GC, McCrimmon RJ, Deary IJ, Frier BM. Perceived symptoms of hypoglycaemia in elderly type 2 diabetic patients treated with insulin. Diabet Med. 1998;15(5):398-401.

27. Lipska KJ, Warton EM, Huang ES, Moffet HH, Inzucchi SE, Krumholz HM, Karter AJ. HbA1c and risk of severe hypoglycemia in type 2 diabetes: the Diabetes and Aging Study. Diabetes Care. 2013;36(11):3535-3542.

28. Miller ME, Bonds DE, Gerstein HC, Seaquist ER, Bergenstal RM, Calles-Escandon J, Childress RD, et al. The effects of baseline characteristics, glycaemia treatment approach, and glycated haemoglobin concentration on the risk of severe hypoglycaemia: post hoc epidemiological analysis of the ACCORD study. BMJ. 2010;340:b5444.

29. Risk of hypoglycaemia in types 1 and 2 diabetes: effects of treatment modalities and their duration. Diabetologia. 2007;50(6):1140-1147.

30. Akram K, Pedersen-Bjergaard U, Borch-Johnsen K, Thorsteinsson B. Frequency and risk factors of severe hypoglycemia in insulin-treated type 2 diabetes: a literature survey. J Diabetes Complications. 2006;20(6):402-408.

31. Schopman JE, Geddes J, Frier BM. Prevalence of impaired awareness of hypoglycaemia and frequency of hypoglycaemia in insulin-treated type 2 diabetes. Diabetes Res Clin Pract. 2010;87(1):64-68.

32. Engel SS, Round E, Golm GT, Kaufman KD, Goldstein BJ. Safety and tolerability of sitagliptin in type 2 diabetes: pooled analysis of 25 clinical studies. Diabetes Ther. 2013;4(1):119-145

33. Rizzo MR, Barbieri M, Boccardi V, Angellotti E, Marfella R, Paolisso G. Dipeptidyl peptidase-4 inhibitors have 
protective effect on cognitive impairment in aged diabetic patients with mild cognitive impairment. J Gerontol A Biol Sci Med Sci. 2014;69(9):1122-1131.

34. Hegazy SK. Evaluation of the anti-osteoporotic effects of metformin and sitagliptin in postmenopausal diabetic women. J Bone Miner Metab. 2015;33(2):207-212.

35. Ikeguchi E, Taniguchi T, Aramaki Y, Araki M, Okamoto M, Hamasaki A. Analysis of Severe Hypoglycemia Leading to Hospitalization in Diabetic Patients Treated with Sulfonylureas or Insulin. J Japan Diabetes Soc. 2014;57(4):235-241.

36. Yabe D, Seino Y. Dipeptidyl peptidase-4 inhibitors and sulfonylureas for type 2 diabetes: Friend or foe? J Diabetes Investig. 2014;5(5):475-477.

37. Oishi M, Yamazaki K, Okuguchi F, Sugimoto H, Kanatsuka A, Kashiwagi A. Changes in oral antidiabetic prescriptions and improved glycemic control during the years 2002-2011 in Japan (JDDM32). J Diabetes Investig. 2014;5(5):581-587.

38. Zhang Y, Wieffer H, Modha R, Balar B, Pollack M,
Krishnarajah G. The burden of hypoglycemia in type 2 diabetes: a systematic review of patient and economic perspectives. JCOM. 2010;17(12):547-557.

39. Cheekati V, Osburne RC, Jameson KA, Cook CB. Perceptions of resident physicians about management of inpatient hyperglycemia in an urban hospital. J Hosp Med. 2009;4(1):E1-8.

40. Zammitt NN, Frier BM. Hypoglycemia in type 2 diabetes: pathophysiology, frequency, and effects of different treatment modalities. Diabetes Care. 2005;28(12):29482961.

41. Murata GH, Duckworth WC, Shah JH, Wendel CS, Hoffman RM. Factors affecting hypoglycemia awareness in insulin-treated type 2 diabetes: The Diabetes Outcomes in Veterans Study (DOVES). Diabetes Res Clin Pract. 2004;65(1):61-67.

42. Zammitt NN, Streftaris G, Gibson GJ, Deary IJ, Frier BM. Modeling the consistency of hypoglycemic symptoms: high variability in diabetes. Diabetes Technol Ther. 2011;13(5):571-578. 